\title{
Neoadjuvant immunotherapy combined with chemotherapy for local advanced non-small cell lung cancer: a case report
}

\author{
Jie Yang ${ }^{1 \#}$, Haoran Xia ${ }^{2 \#}$, Fenghuan Sun ${ }^{1}$, Peng Zhang ${ }^{1}$, Gening Jiang ${ }^{1}$ \\ ${ }^{1}$ Department of Thoracic Surgery, Shanghai Pulmonary Hospital, Tongji University School of Medicine, Shanghai, China; ${ }^{2}$ Medical Graduate School \\ of Nanchang University, Nanchang, China \\ \#These authors contributed equally to this work. \\ Correspondence to: Peng Zhang, MD, PhD; Gening Jiang, MD. Department of Thoracic Surgery, Shanghai Pulmonary Hospital, Tongji University \\ School of Medicine, 507 Zhengmin Road, Shanghai 200433, China. Email: zhangpeng1121@tongji.edu.cn; jgnwp@aliyun.com.
}

\begin{abstract}
Immunotherapy has revolutionized lung cancer management and revitalized the field of tumor immunology. Anti-programmed death 1 (anti-PD-1) immunotherapy can significantly improve the survival of patients with advanced non-small cell lung cancer (NSCLC), but its role in neoadjuvant therapy of local advanced NSCLC remains uncertain. Herein, we reported a case from a clinical trial of our department, a patient who achieved excellent outcome after neoadjuvant immunotherapy and chemotherapy for local advanced NSCLC. A 70 years old male patient with stage IIIA squamous cell carcinoma was admitted in our hospital, who initially needed to undergo pneumonectomy. After two cycles of neoadjuvant immunotherapy combined with chemotherapy, the tumor significantly shrank and the patient underwent left upper lobectomy finally. The patient had grade 2 myelosuppression and recovered after injection with recombinant human granulocyte colony stimulating factor. There was no operation-related complication the patient was discharged uneventfully. The patient received two cycles adjuvant chemotherapy combined with immunotherapy, then immunotherapy alone monthly. The patient was well during the 4-month followup after surgery and would receive immunotherapy till one year after surgery. Our case added evidence of the feasibility and efficacy of neoadjuvant immunotherapy combined with chemotherapy in local advanced NSCLC. Randomized, controlled, multi-center studies are needed to confirm these findings.
\end{abstract}

Keywords: Neoadjuvant immunotherapy; programmed death 1 (PD-1); non-small cell lung cancer (NSCLC); case-report; clinical trial

Submitted Feb 26, 2021. Accepted for publication Apr 22, 2021.

doi: 10.21037/atm-21-1565

View this article at: http://dx.doi.org/10.21037/atm-21-1565

\section{Introduction}

Lung cancer has been the leading cause of cancer-related mortality, and nearly $30-40 \%$ of patients had advanced disease or distant metastases at diagnosis (1). With the development of multimodality therapy, the 5 -year survival rate of patients with stage III non-small cell lung cancer (NSCLC) has increased from 9-24\% (2) to $12-41 \%$ (3), although based on different TNM staging system. A meta-analysis of 15 randomized controlled trials showed that compared with surgery alone neoadjuvant with chemotherapy provided only $5 \%$ of survival advantage at 5 years (4), which was equivalent to that provided by post- operative adjuvant chemotherapy $(5,6)$.

Immunotherapy using anti-programmed death 1 (PD-1) or programmed death ligand 1 (PD-L1) antibodies alone, or combined with chemotherapy, has been proved to provided improved survival in stage IV NSCLC. In resectable lung cancer, neoadjuvant therapy with nivolumab and atezolizumab showed major pathologic response (MPR) rate of $21 \%$ and $45 \%$ respectively, with acceptable toxicity, and no increased operative mortality $(7,8)$. While the role of neoadjuvant with immunotherapy combined with chemotherapy in NSCLC is still not fully understood as there is still paucity of relevant study. Herein, we reported 

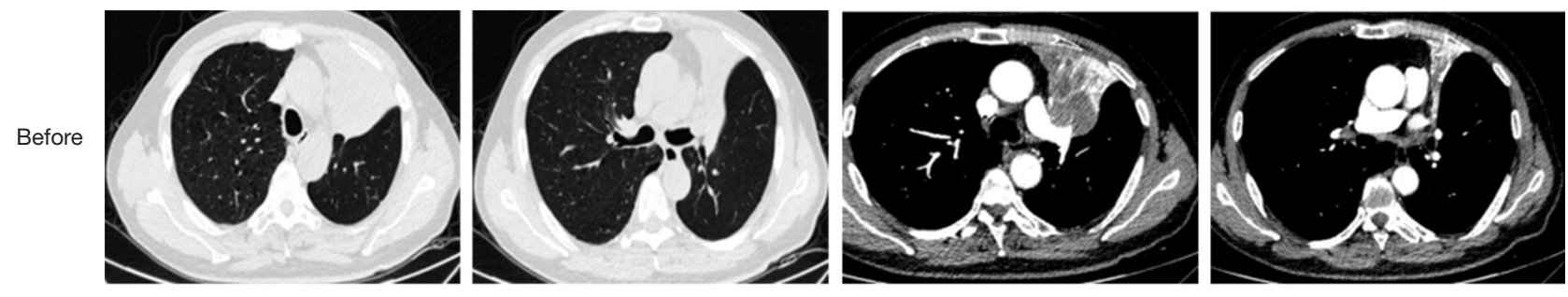

2 cycles neoadjuvant therapy
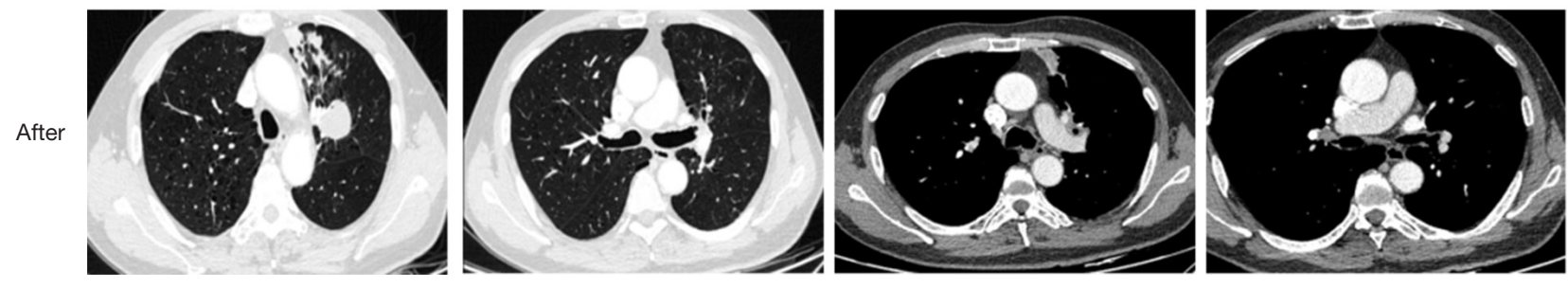

Figure 1 CT scan of the patient before and after neoadjuvant therapy showed the tumor response and remission of the pulmonary atelectasis. CT, computed tomography.

a case from a clinical trial (ChiCTR1900023758), a patient who achieved excellent outcome after neoadjuvant immunotherapy and chemotherapy for local advanced NSCLC. We present the following article in accordance with the CARE reporting checklist (available at http:// dx.doi.org/10.21037/atm-21-1565).

\section{Case presentation}

The present study was approved by the ethical committee board of Shanghai Pulmonary Hospital. All procedures performed in studies involving human participants were in accordance with the ethical standards of the institutional and/or national research committee(s) and with the Helsinki Declaration (as revised in 2013). A 70 years old male patient developed cough and sputum without obvious inducement. Initially, the patient, the patient was treated in other hospital. The computed tomography (CT) scan revealed that patchy opacity in the left upper lobe. Bronchoscopy was performed but didn't succeed because of bleeding. The patient was diagnosed as tuberculosis and was treated with anti-tuberculosis therapy. But there was no significant relief of symptoms after 4 months treatment. Then the patient was transferred to our hospital. Reexamined CT scan showed atelectasis of left upper lobe. Positron-emission tomography-computed tomography (PET-CT) showed a mass about $5 \mathrm{~cm}$ in diameter in the left upper lobe, with SUVmax value of 20.72. Bronchoscopy showed a neoplasm in the left main bronchus, covering the opening of the left upper lobe. The pathological finding of biopsy was NSCLC, tending squamous cell carcinoma. The patient was classified as T2bN2M0, stage IIIA. Multidisciplinary discussion suggested that neoadjuvant therapy should be carried out first, followed by surgical treatment. After the patient signed the informed consent, anti-PD-1 therapy combined with platinum-based chemotherapy was carried out. The anti-PD-1 immunotherapy was carried out with sintilimab $200 \mathrm{mg}$, intravenously, and chemotherapy was carried out with carboplatin AUC5 D1 + gemcitabine $1,000 \mathrm{mg} / \mathrm{m}^{2} \mathrm{D} 1, \mathrm{D} 8$.

During neoadjuvant treatment period, the patient had experienced grade 2 myelosuppression and recovered after injection with recombinant human granulocyte colony stimulating factor. After two cycles neoadjuvant therapy. CT scan showed the tumor response and remission of the pulmonary atelectasis (Figure 1). Bronchoscopy showed that the tumor shrank significantly (Figure 2). Clinical evaluation of the tumor based on response evaluation criteria in solid tumors RECIST version 1.1 showed partially response. Surgery was performed 6 weeks after second cycle of neoadjuvant therapy. The surgical procedure includes left upper lobectomy, pulmonary angioplasty, and mediastinal lymph node dissection. The blood loss during surgery was about $200 \mathrm{~mL}$. Pathology findings showed that the mass is about $4 \mathrm{~cm} \times 3 \mathrm{~cm} \times 3 \mathrm{~cm}$ in size and there were no resident tumor cells. Instead, necrosis and multinucleated 


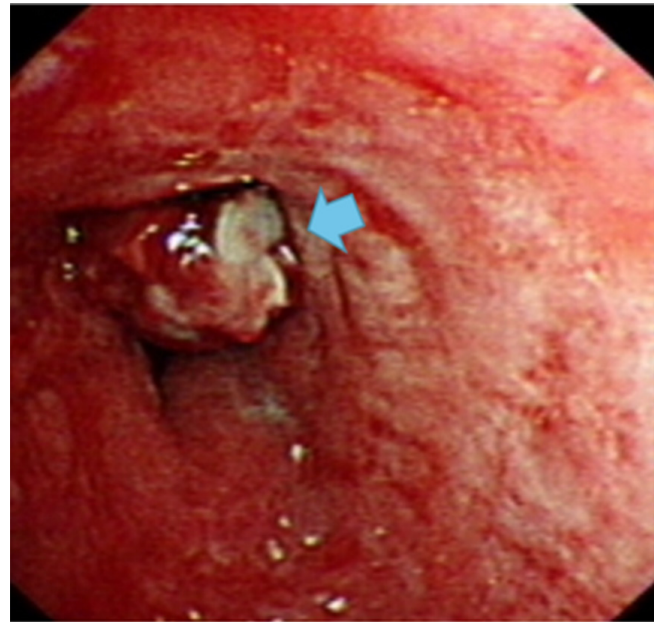

Neoplasm in the left main bronchus

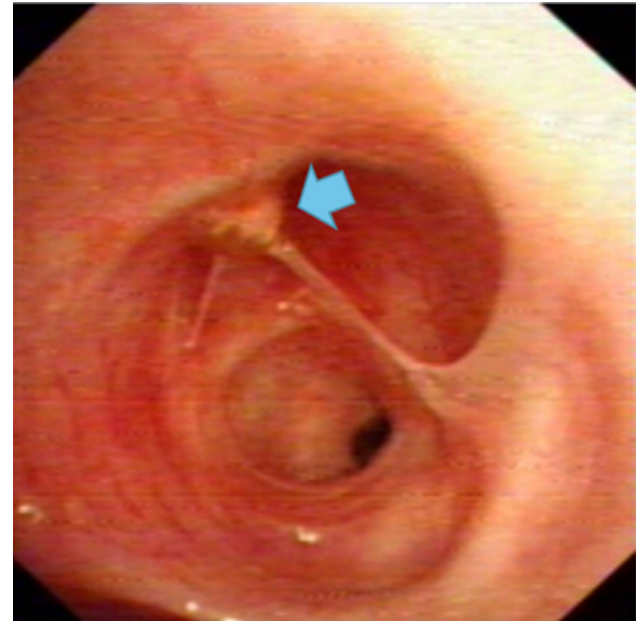

Suspicious neoplasm at the opening of the left upper lobe

Figure 2 Bronchoscopy view of the patient before and after neoadjuvant therapy showed that the tumor shrank significantly. Blue arrows showed the tumor before and after neoadjuvant therapy.

giant cell reaction, cholesterol crystal and fibrous tissue hyperplasia were observed. Pathological findings confirmed pathological complete response (pCR) of the neoadjuvant therapy. Interestingly, a small amount of mycobacterium was found, and tuberculous lesions were considered. All the lymph nodes examined were negative. The patient recovered well and were discharged uneventfully without surgical related complication. As the patient had received anti-tuberculosis treatment for 4 months before the neoadjuvant therapy, and the sputum test for tuberculosis was negative after the operation, considering the tailing effect of immunotherapy and the negative effect of highdose antibiotics on immunotherapy, we decided not to continue the anti-tuberculosis treatment after the operation. The patient received two cycles adjuvant chemotherapy combined with immunotherapy, then immunotherapy alone monthly. The patient was well during the 4-month followup after surgery and would receive immunotherapy till one year after surgery.

\section{Discussion}

Neoadjuvant therapy is the standard therapy for stage IIIA NSCLC. Platinum-based neoadjuvant chemotherapy provides only about $22 \%$ major pathological response (MPR) rate. With the development of immunotherapy, especially anti-PD-1 treatment, more and more thoracic surgeons have paid attention on its role in neoadjuvant therapy. NADIM study (9) showed that the combination of nivolumab, paclitaxel and carboplatin for 3-cycle neoadjuvant therapy resulted in $83 \% \mathrm{MPR}, 59 \% \mathrm{pCR}$, $91 \%$ overall survival (OS) at 18 months, $81 \%$ progression free survival at 18 months, and a $90.2 \%$ down-staging rate, which significantly improved the outcomes of IIIA NSCLC patients with multi-station N2 lymph node metastases.

In the present case, the patient should probably receive pneumonectomy, but after the neoadjuvant therapy, the patient underwent left upper lobectomy instead, which preserved the lung function of the patient and reduced the risk of surgery. The margin was confirmed negative by the frozen section during operation.

As to the safety, the overall incidence of $\mathrm{AE}$ and the incidence of grade 3 and above adverse reactions in immunotherapy were lower than those in chemotherapy $(10,11)$. Combination of anti-PD-1 therapy and platinumbased chemotherapy didn't increase adverse events rate of grade 3 or higher (12). As to the feasibility, pulmonary resection was feasible but cautioned that mediastinal and hilar fibrosis might develop as a result of response to immunotherapy (13). A study showed that resection in these patients was safe and feasible, with perioperative outcomes similar to those in a cohort of patients who received neoadjuvant platinum-based chemotherapy (14). In the present case, considering the difficulty of release of hilum, we performed the surgery by open procedure instead of video-assisted thoracic surgery (VATS).

Previous study showed that the percentages of residual viable tumor cells predict OS and disease-free survival (DFS) 
in patients with resected NSCLC after neoadjuvant (15). In the present case, the patient achieved complete resection and pathological finding confirmed pCR. In most of recent studies, the pathological response rate was used as the primary outcome to evaluate the neoadjuvant therapy. Its impact on long-term survival remains to be further studied.

In conclusion, our case added evidence of the feasibility and efficacy of neoadjuvant immunotherapy combined with chemotherapy in local advanced NSCLC. Randomized, controlled, multi-center studies are needed to confirm these findings.

\section{Acknowledgments}

Funding: This work was supported by Shanghai Hospital Development (Grants No. SHDC2020CR2020B, SHDC12018122), Shanghai Science and Technology Committee (Grant No. 19XD1423200), and Shanghai Pulmonary Hospital (Grants No. fkgg1801, fkcx1904).

\section{Footnote}

Reporting Checklist: The authors have completed the CARE reporting checklist. Available at http://dx.doi.org/10.21037/ atm-21-1565

Conflicts of Interest: All authors have completed the ICMJE uniform disclosure form (available at http://dx.doi. org/10.21037/atm-21-1565). The authors have no conflicts of interest to declare.

Ethical Statement: The authors are accountable for all aspects of the work in ensuring that questions related to the accuracy or integrity of any part of the work are appropriately investigated and resolved. All procedures performed in studies involving human participants were in accordance with the ethical standards of the institutional and/or national research committee(s) and with the Helsinki Declaration (as revised in 2013). Written informed consent was obtained from the patient.

Open Access Statement: This is an Open Access article distributed in accordance with the Creative Commons Attribution-NonCommercial-NoDerivs 4.0 International License (CC BY-NC-ND 4.0), which permits the noncommercial replication and distribution of the article with the strict proviso that no changes or edits are made and the original work is properly cited (including links to both the formal publication through the relevant DOI and the license). See: https://creativecommons.org/licenses/by-nc-nd/4.0/.

\section{References}

1. Herbst RS, Morgensztern D, Boshoff C. The biology and management of non-small cell lung cancer. Nature 2018;553:446-54.

2. Goldstraw P, Crowley J, Chansky K, et al. The IASLC Lung Cancer Staging Project: proposals for the revision of the TNM stage groupings in the forthcoming (seventh) edition of the TNM Classification of malignant tumours. J Thorac Oncol 2007;2:706-14. Erratum in: J Thorac Oncol 2007;2:985.

3. Goldstraw P, Chansky K, Crowley J, et al. The IASLC Lung Cancer Staging Project: Proposals for Revision of the TNM Stage Groupings in the Forthcoming (Eighth) Edition of the TNM Classification for Lung Cancer. J Thorac Oncol 2016;11:39-51.

4. NSCLC Meta-analysis Collaborative Group. Preoperative chemotherapy for non-small-cell lung cancer: a systematic review and meta-analysis of individual participant data. Lancet 2014;383:1561-71.

5. Hellmann MD, Chaft JE, William WN Jr, et al. Pathological response after neoadjuvant chemotherapy in resectable non-small-cell lung cancers: proposal for the use of major pathological response as a surrogate endpoint. Lancet Oncol 2014;15:e42-e50.

6. Arriagada R, Dunant A, Pignon JP, et al. Long-term results of the international adjuvant lung cancer trial evaluating adjuvant Cisplatin-based chemotherapy in resected lung cancer. J Clin Oncol 2010;28:35-42.

7. Blumenthal GM, Bunn PA Jr, Chaft JE, et al. Current Status and Future Perspectives on Neoadjuvant Therapy in Lung Cancer. J Thorac Oncol 2018;13:1818-31.

8. Reck M, Rodríguez-Abreu D, Robinson AG, et al. Pembrolizumab versus Chemotherapy for PD-L1Positive Non-Small-Cell Lung Cancer. N Engl J Med 2016;375:1823-33.

9. Provencio-Pulla M, Nadal-Alforja E, Cobo M, et al. Neoadjuvant chemo/immunotherapy for the treatment of stages IIIA resectable non-small cell lung cancer (NSCLC): A phase II multicenter exploratory study-NADIM studySLCG. J Clin Oncol 2018;36:abstr 8521.

10. Horn L, Spigel DR, Vokes EE, et al. Nivolumab Versus Docetaxel in Previously Treated Patients With Advanced Non-Small-Cell Lung Cancer: Two-Year Outcomes From Two Randomized, Open-Label, Phase III Trials 
(CheckMate 017 and CheckMate 057). J Clin Oncol 2017;35:3924-33.

11. Herbst RS, Baas P, Kim DW, et al. Pembrolizumab versus docetaxel for previously treated, PD-L1-positive, advanced non-small-cell lung cancer (KEYNOTE-010): a randomised controlled trial. Lancet 2016;387:1540-50.

12. Gandhi L, Rodríguez-Abreu D, Gadgeel S, et al.

Pembrolizumab plus Chemotherapy in Metastatic NonSmall-Cell Lung Cancer. N Engl J Med 2018;378:2078-92.

13. Chaft JE, Hellmann MD, Velez MJ, et al. Initial Experience With Lung Cancer Resection After Treatment With T-Cell Checkpoint Inhibitors. Ann Thorac Surg

Cite this article as: Yang J, Xia H, Sun F, Zhang P, Jiang G. Neoadjuvant immunotherapy combined with chemotherapy for local advanced non-small cell lung cancer: a case report. Ann Transl Med 2021;9(8):724. doi: 10.21037/atm-21-1565 2017;104:e217-e218.

14. Yang CJ, McSherry F, Mayne NR, et al. Surgical Outcomes After Neoadjuvant Chemotherapy and Ipilimumab for Non-Small Cell Lung Cancer. Ann Thorac Surg 2018;105:924-9.

15. Pataer A, Kalhor N, Correa AM, et al. Histopathologic response criteria predict survival of patients with resected lung cancer after neoadjuvant chemotherapy. J Thorac Oncol 2012;7:825-32.

(English Language Editor: J. Teoh) 